\title{
Mid- to Long-term Outcomes After Weber B-type Ankle Fractures With and Without Syndesmotic Rupture
}

\author{
MICHAEL B.J. KOHAKE ${ }^{1,2}$, ULRICH WIEBKING ${ }^{1,2}$, PADHRAIG F. O'LOUGHLIN ${ }^{3}$, \\ CHRISTIAN KRETTEK ${ }^{2}$ and RALPH GAULKE ${ }^{1,2}$ \\ ${ }^{1}$ Section Upper Extremity, Foot- and Rheuma Surgery, Trauma Department, \\ Medical School Hanover (MHH), Hanover, Germany; \\ ${ }^{2}$ Trauma Department, Medical School Hanover (MHH), Hanover, Germany; \\ ${ }^{3}$ Mater Hospital, City Gate Mahon, Cork, Ireland
}

\begin{abstract}
Background/Aim: The aim of the present study was to assess the impact of syndesmotic screw fixation on overall clinical outcomes following Weber B-type ankle fractures. Materials and Methods: A total of 21 patients with syndesmotic rupture requiring screw fixation were compared to 40 patients with an intact syndesmosis. Olerud-Molander-Ankle-Score, American Orthopedic Foot \& Ankle Society ankle hindfoot score, and the Short Form Health Survey-36 were recorded. Weight-bearing plain radiographs were performed to rate post traumatic osteoarthrosis according to the Kellgren-Lawrence score. Pain levels were evaluated with a visual analog scale. Results: A total of 61 patients with a mean follow-up of 6.6 years (range $=2-12$ years) satisfied the inclusion criteria. Pain level, clinical outcome scores, and radiographs did not reveal significant differences between the groups. Ankle joints with syndesmotic rupture showed a significant restriction in dorsiflexion compared to those with an intact syndesmosis (15 vs. $\left.20^{\circ}, p=0.028\right)$. Conclusion: Syndesmotic rupture does not affect clinical and radiological outcome parameters following Weber B-type ankle fractures, but does lead to a significant restriction in dorsiflexion of the ankle joint.
\end{abstract}

Weber B-type ankle fractures are common and, if displaced, are usually treated with open reduction and internal fixation (1-3). In more than one third of cases, the distal tibiofibular

This article is freely accessible online.

Correspondence to: Professor Dr. med. Ralph Gaulke, Section Upper Extremity, Foot- and Rheuma Surgery, Trauma Department, Medical School Hanover (MHH), Carl-Neuberg-Str. 1, D-30625 Hannover, Germany. Tel: +49 5115322015, e-mail: gaulke.ralph@mhhannover.de

Key Words: Syndesmotic rupture, ankle fracture, Weber B-type, mid-to-long-term follow-up. syndesmosis is disrupted (4) which may result in an unstable ankle, post traumatic osteoarthrosis and worse clinical outcomes $(5,6)$. Typically, syndesmotic screws are used to restore ankle joint stability and prevent poor functional results as well as early osteoarthrosis (7-9) (Figure 1).

The effect of syndesmotic injury on patient outcomes remains somewhat controversial. Several studies have found similar functional and radiological results in patients who sustained an ankle fracture with or without syndesmotic instability $(10,11)$. However, Egol et al. reported inferior ankle function in patients after Weber A-C-type ankle fractures and associated syndesmotic injury compared to patients with a stable syndesmosis and bony fixation alone (12). To the current authors' knowledge Weber B-type ankle fractures in which syndesmotic injuries were consistently treated with syndesmotic screw fixation have not been studied previously.

The aim of the present study was to assess the impact of syndesmotic screw fixation on overall clinical outcomes following Weber B-type ankle fractures. Mid- to long-term radiological and functional results of patients with syndesmotic injury and screw fixation were compared to those of patients without syndesmotic injury.

\section{Materials and Methods}

This was a retrospective clinical and radiological trial examining the effect of a syndesmotic injury on the outcome of Weber B-type ankle fractures. The study protocol was approved by the local Ethics and Research Committee (No. 7520) and is registered with the Deutsches Register für klinische Studien ("German Clinical Trials Register") as DRKS00012838.

All adult patients suffering from isolated, closed Weber B-type ankle fractures, requiring surgical intervention at the authors' university hospital between January 2006 and July 2016, were included in the study. Exclusion criteria were open fractures, dementia, additional fractures of the medial malleolus, patients with multiple injuries and fracture fixation revision. The patients were grouped either to syndesmotic injury and screw fixation or intact syndesmosis, with a minimum of two years of follow-up. 

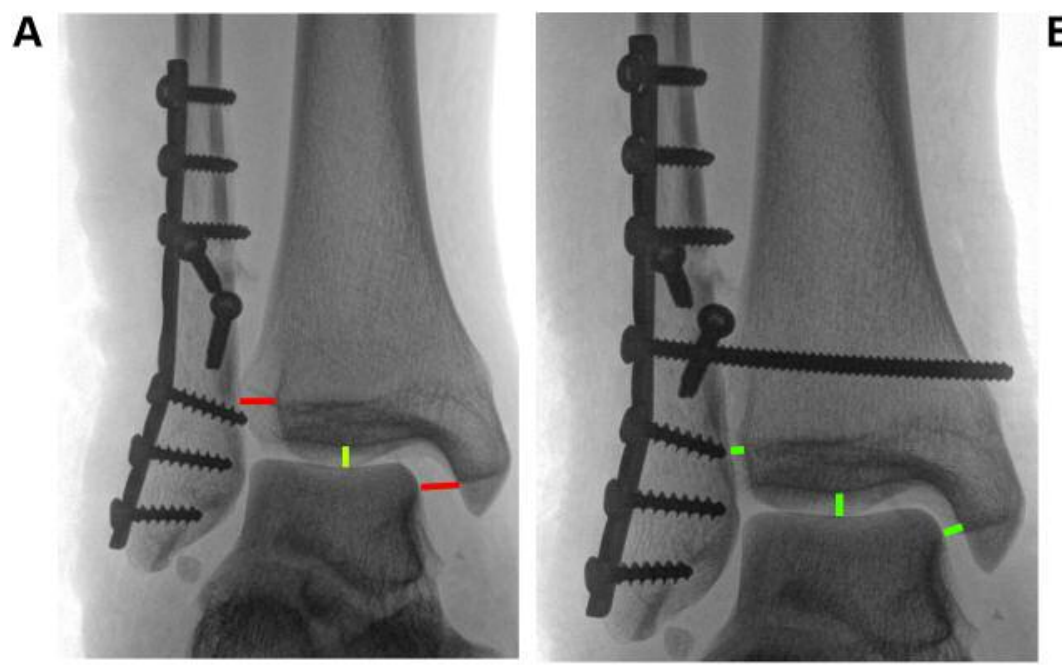

Figure 1. Distal fibula fracture (A) after bony fixation with insufficient reduction of the tibiofibular syndesmosis: red lines show widening of the tibiofibular syndesmosis and the medial joint space. The horizontal tibiotalar joint space is normal (green line). (B) after reduction and fixation with an additional syndesmotic screw: distal tibiofibular distance and joint spaces are normal (green lines).

Table I. Kellgren-Lawrence score.

\begin{tabular}{lcc}
\hline Grade 0 & 0 points & Definite absence of osteoarthrotic changes \\
Grade 1 & $1-2$ points & Doubtful JSN*, possible osteophytic lipping \\
Grade 2 & $3-4$ points & Definite osteophytes, possible JSN \\
Grade 3 & $5-9$ points & Moderate osteophytes, definite JSN, some sclerosis, possible epiphyseal deformity \\
Grade 4 & 10 points & Large osteophytes, marked JSN, severe sclerosis, definite epiphyseal deformity \\
\hline
\end{tabular}

*JSN: Joint space narrowing.

Senior orthopedic foot and ankle surgeons performed the surgery. Stability of the syndesmosis was evaluated intraoperatively using the hook test (Figure 2). The fibula was pulled laterally using a bone hook to evaluate the stability of the tibiofibular syndesmosis $(13,14)$. Widening of more than $2 \mathrm{~mm}$ was defined as an indirect sign of syndesmotic rupture. In cases of an unstable distal fibula, fixation was achieved with a screw parallel to the syndesmosis thus approximating the path of the tibiofibular syndesmosis.

All patients were examined by MK under the supervision of RG und UW. Range-of-motion (ROM) of the ankle joint was measured between the plantar aspect of the hind foot and the fibula using a goniometer. Ankle and subtalar stability and motion as well as pain to pressure and translational stress on the joints of the hindfoot were measured. Clinical examination also included measurement of the hind foot axis whilst weightbearing, scar assessment, evaluation of skin vascularity and sensitivity to touch. Semi-quantitative measurement of weight distribution at the plantar aspect of the foot was performed on a mirror-table (Figure 3). The operated side was compared to the contralateral uninjured foot.

The primary outcome measures of this study were OlerudMolander-Ankle-Score (OMAS) (15), Hannover-Score (HS), FootFunction-Index (FFI) and American Orthopedic Foot and Ankle Society (AOFAS) scoring system. The Short Form Health Survey
(SF)-36 v2.0 was used to evaluate limitations in health-related quality of life. Study participants completed the questionnaires during the follow-up visit at the authors' hospital.

Postoperative osteoarthrosis of the ankle joint was evaluated using the Kellgren-Lawrence Score (KLS) (Table I) (16) on lateral and mortise view radiographs of the weight bearing foot. KLS grade higher than "1" was defined as significant osteoarthrosis. The posttraumatic increase in KLS was documented. All radiographs were scored by MK and UW.

Statistical analysis. To compare the baseline characteristics as well as functional and mental outcome parameters of both groups, a $t$-test for two independent samples was performed. Two-tailed $p$-values and $95 \%$ confidence intervals $(95 \% \mathrm{CIs})$ are presented. Cross tables with Fisher exact test were used to evaluate nominal and ordinal data. All data were analyzed using SPSS 25. $p$-Values less than 0.05 were considered significant.

\section{Results}

Enrolment and patient details. Between January 2006 and July 2016, 176 out of 404 patients with Weber B-type ankle fractures met the inclusion criteria. A total of 65 patients 


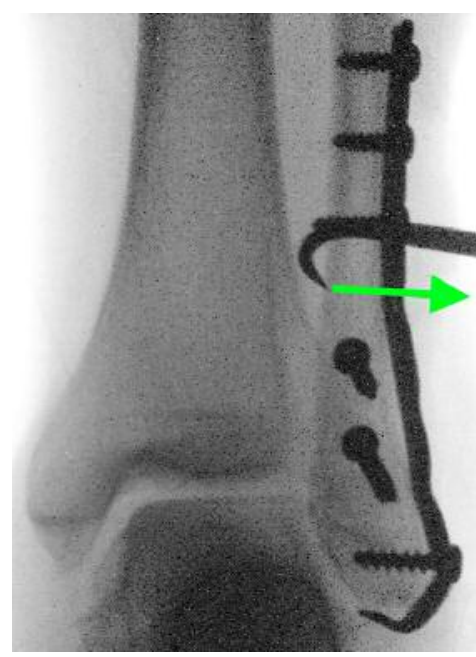

Figure 2. Intraoperative Hook test to test the stability of the syndesmosis following osteosynthesis of the distal fibula fracture.

Table II. Baseline characteristics.

\begin{tabular}{lccc}
\hline & Screw & No-Screw & $p$-Value \\
\hline Patients & 21 & 40 & \\
Mean agea ${ }^{\text {, years (SD) }}$ & $59(16)$ & $54(17)$ & 0.273 \\
Gender & & & \\
$\quad$ M & 11 & 21 & 0.993 \\
F & 10 & 19 & \\
Follow-up, years (SD) & $6(3)$ & $7(3)$ & 0.188 \\
BMIb (SD) & $28(6)$ & $26(4)$ & 0.168 \\
Fracture type & & & \\
$\quad$ Weber B & 21 & 40 & \\
$\quad$ Syndesmotic rupture & 21 & 0 & \\
Anatomy of fracture & & & \\
$\quad$ Fibula & 12 & 39 & $>0.001$ \\
Fibula + post malleolus & 9 & 1 & \\
Fibula + med malleolus & 0 & 0 & 0.844 \\
$\quad$ Open fracture & 0 & 0 & \\
$\quad$ Ankle ligament injuryc & 3 & 5 & \\
\hline
\end{tabular}

${ }^{\mathrm{a} A g e}$ at time of follow-up; ${ }^{\mathrm{b}}$ Body mass index at time of surgery; ${ }^{\mathrm{c}}$ Ankle ligament injury including deltoid ligament and fibular ligaments. Post malleolus: Tibial insertion of the dorsal tibiofibular syndesmosis; BMI Body mass index; SD: standard deviation.

were lost to follow-up; 47 patients refused the invitation for examination; two had died and one patient underwent a lower leg amputation that was not due to the ankle fracture. Overall, 21 patients with and another 40 without syndesmotic injury participated in the clinical examination with a mean of 6.6 years (range $=2-12$ years) of follow-up. In 58 out of these 61 patients, plain radiographs were performed.

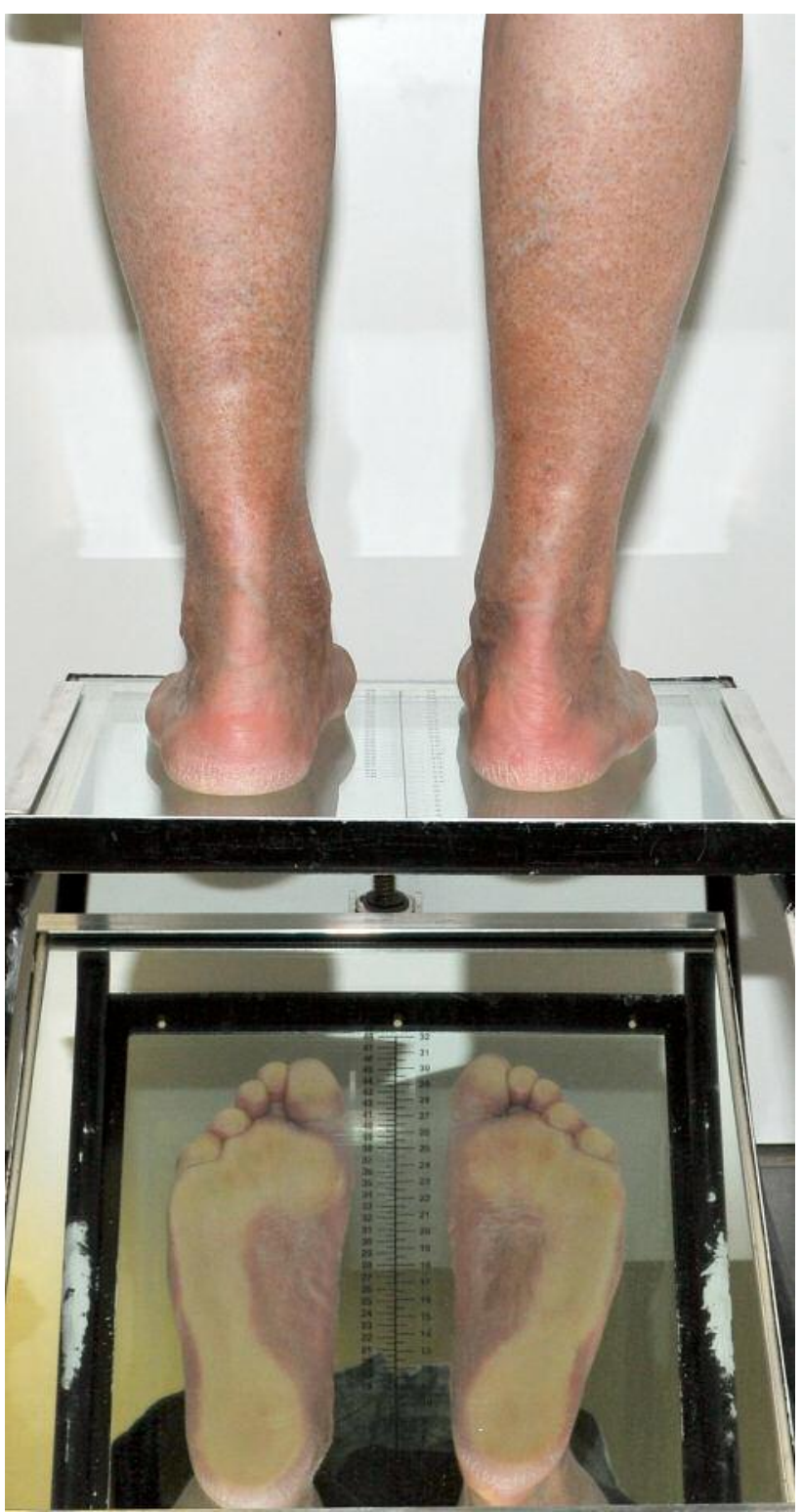

Figure 3. Semi-quantitative analysis of plantar weight-bearing pressure distribution using a mirror-table.

The two groups did not differ significantly in gender distribution, age, follow-up period or body mass index (Table II). There was a greater percentage of patients with an additional fracture of the tibial insertion of the dorsal syndesmosis in the syndesmotic injury group $(p<0.001)$. There was no difference in the incidence of an additional ankle ligament injury between the groups $(p=0.844)$.

Patient outcome scores. Patients with healed, previously ruptured and those with a primary intact syndesmosis had 
Table III. Functional, mental and pain outcome of screw and no-screw group.

\begin{tabular}{|c|c|c|c|c|c|c|}
\hline & Screw, mean & $\mathrm{SD}$ & No-Screw, mean & $\mathrm{SD}$ & $95 \%$ CI of Difference & $p$-Value \\
\hline OMAS & 84 & 20 & 90 & 17 & -3 to 16 & 0.178 \\
\hline AOFAS-AHS & 85 & 12 & 89 & 11 & -2 to 10 & 0.170 \\
\hline VAS, morning & 0.3 & 0.8 & 0.5 & 1.0 & -0.3 to 0.7 & 0.463 \\
\hline VAS, evening & 1.2 & 2.0 & 1.1 & 1.2 & -1.0 to 0.8 & 0.843 \\
\hline SF-36 PCS & 51 & 9 & 54 & 9 & -2 to 7 & 0.317 \\
\hline SF-36 MCS & 51 & 10 & 52 & 7 & -4 to 5 & 0.871 \\
\hline Dorsiflexion, degree & 15 & 6 & 20 & 8 & 1 to 9 & 0.028 \\
\hline Plantarflexion, degree & 28 & 7 & 30 & 8 & -2 to 7 & 0.324 \\
\hline FFI, pain ${ }^{\mathrm{a}}$ & 8 & 11 & 5 & 7 & -7 to 2 & 0.237 \\
\hline FFI, function ${ }^{b}$ & 14 & 16 & 7 & 12 & -14 to 0 & 0.053 \\
\hline Hannover-score ${ }^{c}$ & 37 & 15 & 30 & 13 & -14 to 1 & 0.072 \\
\hline
\end{tabular}

a,b ${ }^{2}$ ower values indicate less pain and impairment in function (scale 0 to 100); cLower values indicate less impairment in function (scale 20-100). SD: Standard deviation; CI: confidence interval; OMAS: Olerud-Molander-Ankle-Score; AOFAS-AHS: American Orthopedic Foot \& Ankle Society ankle hindfoot score; VAS: Visual analog scale; SF-36: Short Form Health Survey-36; PCS: Physical Component Summary; MCS: Mental Component Summary; FFI: Foot-Function-Index.

similar results in FFI, AOFAS and Hannover-Score. There was a trend in the OMAS towards superior outcome in ankles with an intact syndesmosis (90 vs. 84, $p=0.178$ ). Plantarflexion did not differ between the groups. SF-36 Physical and Mental Component Summary (PCS, MCS) as well as SF-36 subscales were similar in both groups. No differences were observed in VAS scores between patients with or without syndesmotic rupture with regard to pain in the morning and at the end of the day $(0.3$ vs. $0.5, p=0.463$; 1.2 vs. $1.1, p=0.843)$. However, patients with a syndesmotic injury experienced a significant restriction in dorsiflexion of the ankle joint with a mean difference of 5 degrees $\left(15^{\circ}\right.$, range 0 to $25^{\circ}, v s .20^{\circ}$, range 0 to $40^{\circ}, p=0.028$ ) (Table III).

Weight-bearing analysis. Valgus malalignment of the normal hindfoot axis in $5(8 \%)$ patients as well as pes planus in 7 (12\%) patients were found. In addition, 6 (10\%) patients had a combination of both. Furthermore, pes cavus was detected in $5(8 \%)$ patients and splayfoot in $4(7 \%)$ patients. Isolated cases of pes planus and splayfoot were bilateral. Thus, these changes were most likely preexisting and not secondary to injury. In contrast, a hindfoot valgus, the combination of hindfoot valgus and pes planus as well as an isolated pes cavus were found in $50 \%$ of patients ( 8 out of 16) only on the treated side. However, the incidence of unilateral changes of the plantar aspect of the weight-bearing foot, was not significantly different between patients with and without syndesmotic injury ( $18 \%$ vs. $11 \%, p=0.703)$.

Radiographic analysis. At follow-up, 11 out of 58 ankles (19\%) showed no sign of osteoarthrosis (OA). A total of 31 ankles $(53 \%)$ were scored as KLS grade 1, 14 (24\%) as grade 2 , two (4\%) as grade 3 and none as grade 4 . Eleven
Table IV. Radiographic outcome of the ankle joint.

\begin{tabular}{lccc}
\hline & Screw & No-screw & $p$-Value \\
\hline Normal joint & $2(6)$ & $9(15)$ & 0.196 \\
KLS grade 1 & $12(12)$ & $19(20)$ & \\
KLS grade 2 & $5(3)$ & $9(2)$ & \\
KLS grade 3 & $2(0)$ & $0(0)$ & \\
KLS grade 4 & $0(0)$ & $0(0)$ & \\
OA total (KLS grade $\geq 2)$ & $7(3)$ & $9(2)$ & 0.546 \\
KLS increase +1 & 11 & 8 & 0.701 \\
KLS increase +2 & 1 & 1 & \\
\hline
\end{tabular}

aRadiographs are missing from 3 patients; ${ }^{b}$ chi-square with Fisher exact test; cincrease of KLS between time of surgery and follow-up. Number of patients with preexisting osteoarthrosis at time of ankle surgery are shown in parentheses. KLS: Kellgren-Lawrence-Score; OA: osteoarthrosis.

(28\%) patients in the group with intact syndesmosis and 8 (38\%) patients in the group with syndesmotic rupture showed a slight increase in KLS (+1) after surgery. In addition, a moderate increase (KLS +2) was found in a single patient in both groups. No significant differences between groups were found regarding the KLS at time of follow-up $(p=0.196)$, the incidence, or the increase of OA after surgery $(p=0.701)$ (Table IV).

Influence of implant removal. In all but one patient with syndesmotic injury, the syndesmotic screw was removed after a minimum of six weeks postoperatively. Two of 21 patients had concomitant removal of all hardware. Another 9 patients underwent removal of all hardware at a later time point. 19 out of 40 patients with intact syndesmosis underwent implant removal. The incidence of complete implant removal was not 
Table V. Outcome after implant removal vs. implant in situ.

\begin{tabular}{|c|c|c|c|c|c|c|}
\hline & Implant removal, mean & SD & No removal, mean & SD & $95 \%$ CI of Difference & $\mathrm{p}$-Value \\
\hline OMAS & 91 & 16 & 90 & 18 & -11 to 11 & 0.958 \\
\hline AOFAS-AHS & 90 & 11 & 89 & 11 & -9 to 6 & 0.665 \\
\hline VAS, morning & 0.5 & 1 & 0.6 & 1 & -0.6 to 0.8 & 0.770 \\
\hline VAS, evening & 1.1 & 1.0 & 1.1 & 1.1 & -0.9 to 1.1 & 0.850 \\
\hline SF-36 PCS & 54 & 9 & 53 & 9 & -6 to 6 & 0.939 \\
\hline SF-36 MCS & 53 & 6 & 50 & 8 & -7 to 2 & 0.251 \\
\hline Dorsiflexion, degree & 18 & 8 & 21 & 8 & -2 to 8 & 0.227 \\
\hline Plantarflexion, degree & 31 & 8 & 29 & 9 & -7 to 3 & 0.458 \\
\hline FFI, pain ${ }^{a}$ & 5 & 8 & 5 & 7 & -5 to 5 & 0.919 \\
\hline FFI, function ${ }^{b}$ & 7 & 11 & 7 & 13 & -7 to 8 & 0.932 \\
\hline Hannover-Score ${ }^{c}$ & 31 & 13 & 30 & 14 & -10 to 7 & 0.791 \\
\hline
\end{tabular}

a,b Lower values indicate less pain and impairment in function (scale 0 to 100); cLower values indicate poorer function (scale 20-100). SD: Standard deviation; CI: confidence interval; OMAS: Olerud-Molander-Ankle-Score; AOFAS-AHS: American Orthopedic Foot \& Ankle Society ankle hindfoot score; VAS: visual analog scale; SF-36: Short Form Health Survey-36; PCS: Physical Component Summary; MCS: Mental Component Summary; FFI: Foot-Function-Index.

significantly different between the groups $(p=0.791)$. Patients with and without hardware removal experienced similar outcomes with regard to functional, radiological and mental outcome scores (Table V).

Postoperative complications and additional surgery. Broken $(\mathrm{n}=2)$ and loose $(\mathrm{n}=2)$ syndesmotic screws were noted. Two patients in each group had a postoperative wound infection $(p=0.602)$. Wound healing problems were found in 6 patients, 2 after syndesmotic injury and 4 with an intact syndesmosis $(p=0.169)$. One patient needed negative pressure wound therapy. No patients needed arthrolysis, arthrodesis or ankle joint replacement.

\section{Discussion}

The aim of the present study was to assess the impact of syndesmotic screw fixation on overall clinical outcomes following Weber B-type ankle fractures. Mid- to long-term radiological and functional results of patients with syndesmotic injury and screw fixation were compared to those of patients without syndesmotic injury. Neither functional outcome scores, pain levels nor SF-36 PCS and MCS were significantly different. The likelihood of posttraumatic OA was not different in patients with or without syndesmotic injury. Both groups experienced restoration of ankle function with good-to-excellent results in $92 \%$ of patients, according to OMAS. However, ankle joints with syndesmotic rupture showed a significant restriction in dorsiflexion compared to those with an intact syndesmosis (15- vs. $\left.20^{\circ}, p=0.028\right)$. Based on the current study's findings, syndesmotic injuries treated with tibiofibular screw fixation, do not have a significant effect on the clinical outcome or quality of life of patients undergoing surgery for a type Weber B ankle fracture.
With regard to previous studies, OMAS and AOFAS values are reported to range from 74 to 93 and 83 to 96 respectively, in patients following ankle fractures $(10,11,17$ 22). The current study cohort generated comparable OMAS and AOFAS values of $88(\mathrm{SD} \pm 18)$ and $88(\mathrm{SD} \pm 11)$ respectively, at two to twelve years follow-up.

In the current literature, various radiological scoring systems $(16,23)$ were used to grade osteoarthrosis after ankle trauma surgery. Therefore, it is difficult to compare the results. The current authors defined $\mathrm{OA}$ as the presence of osteophytes with joint space narrowing and/or deformation. According to this definition, Holzer et al. found OA in $27.4 \%$ of Weber B- and C-type ankle fractures after 18 years (18), whereas other studies reported OA in 6-17\% of patients in Weber B-type ankle fractures after 4-6 years of follow-up $(10,11)$. In the current study population, $28 \%$ of patients developed $\mathrm{OA}$ at 6.6 years follow-up. No significant differences between the treatment groups were found.

Some earlier studies (10-12) evaluated the effect of syndesmotic injury in ankle fractures, but only one study involved a comparable study design to the current study (10). Kortekangas et al. prospectively examined the effect of screw fixation for syndesmotic injury in 48 patients with Weber B-type ankle fractures after a minimum follow-up of four years. The syndesmotic injury was either treated with or without repair of the syndesmosis. OMAS value as well as pain levels and health-related quality of life-score did not demonstrate any significant difference between the treatment groups (10). These findings are similar to the results of the present study. Veen et al. reviewed 59 patients with or without syndesmotic injury with a mean follow-up of 6.8 years postoperatively. The study population consisted of 19 Weber B- and 40 Weber C-type ankle fractures. Similar to the current study's results, they reported no significant 
differences between patients with and without syndesmotic rupture with regard to both OMAS and AOFAS-AHS (11).

There was only one study that found inferior functional outcomes in patients with syndesmotic injury compared to those with an intact syndesmosis. Egol et al. examined 347 patients with all Weber types of ankle fractures, after 6 and 12 months of follow-up. Weber C-type ankle fractures were associated more frequently with syndesmotic injury than Weber B-type ankle fractures. Patients with syndesmotic fixation generated lower values in the AOFAS scoring system and reported less pain (12). This pattern of results may be attributable to a relatively short follow-up interval and/or the heterogeneity of fracture types included in the study. With regard to the current literature, the Weber C-type ankle fracture represents a higher severity of ankle injury and leads to a poorer radiological and clinical outcome (18). On the other hand, a larger study population increased the likelihood of establishing statistically significant results.

In contrast to all of the other outcome parameters in the current study, the authors did detect a difference in post-op dorsiflexion. There were significantly lower values in dorsiflexion (mean $5^{\circ}$ ) amongst ankle joints treated with indirect screw fixation of the distal tibiofibular joint. Several similar studies reported no difference in the ROM of fractured ankles with and without concomitant syndesmotic rupture $(10,12)$. However, differing measurement techniques for ROM, variations in physical therapy protocols and heterogeneity in terms of the fracture types studied, may explain these results.

Considering earlier studies $(10,11)$ as well as the results of the present investigation, syndesmotic injury, treated with tibiofibular screw fixation, does not seem to affect patients' subjective and objective outcomes after Weber B-type ankle fracture in mid- to long-term follow-up.

Hardware removal accounts for $29 \%$ of all elective operations in trauma units (24). Several studies examined the effect of screw or plate removal on pain level and functional outcomes $(12,17,20,25)$. Jung et al. found decreased pain levels and improvements in ankle stiffness in patients who underwent implant removal (26). In contrast, Brown et al. found decreased pain after hardware removal in only $50 \%$ of patients and no differences in function and quality of life after hardware removal compared to those who left the hardware in situ (27). Moreover, a majority of surgeons did not agree that routine implant removal is necessary and rated the effectiveness of implant removal in symptomatic patients as only moderate (28). In the current study, patients with plate removal did not generate superior functional and radiological results. Therefore, implant removal as a routine procedure does not seem to improve clinical outcomes. Leaving hardware in situ could reduce a significant burden on hospital resources without impacting negatively on patients (24).
The current study has inherent limitations including its retrospective design. Moreover, due to the lengthy recruitment period, operations were performed by various surgeons. The clinical results of patients with very different follow-up periods were combined, which may have biased the findings; although mean follow-up time did not differ between the groups. The strengths of the present study are the assessment of patient outcomes with widely-used and validated outcome scores and the precise inclusion and exclusion criteria. In earlier studies, the effect of a syndesmotic injury may have been difficult to ascertain when pooling different fracture types into one group and if patients with additional injuries such as the fractures of the medial malleolus were included.

\section{Conclusion}

Rupture of the tibiofibular syndesmosis, treated with screw fixation, does not have a negative impact on the clinical and radiological mid- to long-term outcomes of Weber B-type ankle fractures despite a mild limitation of ankle dorsiflexion.

\section{Acknowledgements}

The present study was financially supported by the Klin-StrucMed program of the independent Else-Kröner-Foundation (Else-KrönerStiftung) for the clinical MD of MK.

\section{References}

1 Goost H, Wimmer MD, Barg A, Kabir K, Valderrabano V and Burger C: Fractures of the Ankle Joint: Investigation and Treatment Options. Dtsch Arztebl Int 111(21): 377-388, 2014.

2 Deutsche Gesellschaft für Unfallchirurgie e V: Sprunggelenkfraktur. Available at: https://www.awmf.org/ uploads/tx_szleitlinien/0120031_S2e_Sprunggelenkfraktur_ 2016-02.pdf, 2015. Accessed 31.07.2018.

3 Jensen SL, Andresen BK, Mencke S and Nielsen PT: Epidemiology of ankle fractures. A prospective population-based study of 212 cases in Aalborg, Denmark. Acta Orthop Scand 69(1): 48-50, 1998.

4 Stark E, Tornetta P and Creevy WR: Syndesmotic instability in Weber B ankle fractures: a clinical evaluation. J Orthop Trauma 21(9): 643-646, 2007.

5 Lloyd J, Elsayed S, Hariharan K and Tanaka H: Revisiting the concept of talar shift in ankle fractures. Foot Ankle Int 27(10): 793-796, 2006

6 Still GP and Atwood TC: Operative outcome of 41 ankle fractures: a retrospective analysis. J Foot Ankle Surg 48(3): 330339, 2009.

7 Pettrone FA, Gail M, Pee D, Fitzpatrick T and Van Herpe LB: Quantitative criteria for prediction of the results after displaced fracture of the ankle. J Bone Joint Surg Am 65(5): 667-677, 1983.

8 Schnetzke M, Vetter SY, Beisemann N, Swartman B, Grützner PA and Franke J: Management of syndesmotic injuries: What is the evidence? World J Orthop 7(11): 718-725, 2016. 
9 Yuen CP and Lui TH: Distal Tibiofibular Syndesmosis: Anatomy, Biomechanics, Injury and Management. Open Orthop J 11: 670-677, 2017.

10 Kortekangas T, Flinkkila T, Niinimaki J, Lepojarvi S, Ohtonen $\mathrm{P}$, Savola $\mathrm{O}$ and Pakarinen $\mathrm{H}$ : Effect of syndesmosis injury in SER IV (Weber B)-type ankle fractures on function and incidence of osteoarthritis. Foot Ankle Int 36(2): 180-187, 2015.

11 Veen EJ and Zuurmond RG: Mid-term results of ankle fractures with and without syndesmotic rupture. Foot Ankle Surg 21(1): 30-36, 2015

12 Egol KA, Pahk B, Walsh M, Tejwani NC, Davidovitch RI and Koval KJ: Outcome after unstable ankle fracture: effect of syndesmotic stabilization. J Orthop Trauma 24(1): 7-11, 2010.

13 Stoffel K, Wysocki D, Baddour E, Nicholls R and Yates P: Comparison of two intraoperative assessment methods for injuries to the ankle syndesmosis. A cadaveric study. J Bone Joint Surg Am 91(11): 2646-2652, 2009.

14 Jenkinson RJ, Sanders DW, Macleod MD, Domonkos A and Lydestadt J: Intraoperative diagnosis of syndesmosis injuries in external rotation ankle fractures. J Orthop Trauma 19(9): 604609,2005

15 Olerud $\mathrm{C}$ and Molander $\mathrm{H}$ : A scoring scale for symptom evaluation after ankle fracture. Arch Orthop Trauma Surg 103(3): 190-194, 1984.

16 Kellgren JH and Lawrence JS: Radiological assessment of osteoarthrosis. Ann Rheum Dis 16(4): 494-502, 1957.

17 Hamid N, Loeffler BJ, Braddy W, Kellam JF, Cohen BE and Bosse MJ: Outcome after fixation of ankle fractures with an injury to the syndesmosis: the effect of the syndesmosis screw. J Bone Joint Surg Br 91(8): 1069-1073, 2009.

18 Holzer N, Salvo D, Marijnissen AC, Vincken KL, Ahmad AC, Serra E, Hoffmeyer P, Stern R, Lübbeke A and Assal M: Radiographic evaluation of posttraumatic osteoarthritis of the ankle: the Kellgren-Lawrence scale is reliable and correlates with clinical symptoms. Osteoarthritis Cartilage 23(3): 363-369, 2015.

19 Stufkens SA, Knupp M, Lampert C, van Dijk CN and Hintermann B: Long-term outcome after supination-external rotation type-4 fractures of the ankle. J Bone Joint Surg Br 91(12): 1607-1611, 2009.
20 Weening B and Bhandari M: Predictors of functional outcome following transsyndesmotic screw fixation of ankle fractures. $\mathrm{J}$ Orthop Trauma 19(2): 102-108, 2005.

21 Hoiness $\mathrm{P}$ and Stromsoe K: Tricortical versus quadricortical syndesmosis fixation in ankle fractures: a prospective, randomized study comparing two methods of syndesmosis fixation. J Orthop Trauma 18(6): 331-337, 2004.

22 Wikeroy AK, Hoiness PR, Andreassen GS, Hellund JC and Madsen JE: No difference in functional and radiographic results 8.4 years after quadricortical compared with tricortical syndesmosis fixation in ankle fractures. J Orthop Trauma 24(1): 17-23, 2010.

23 van Dijk CN, Verhagen RA and Tol JL: Arthroscopy for problems after ankle fracture. J Bone Joint Surg Br 79(2): 280-284, 1997.

24 Bostman $\mathrm{O}$ and Pihlajamaki H: Routine implant removal after fracture surgery: a potentially reducible consumer of hospital resources in trauma units. J Trauma 41(5): 846-849, 1996.

25 Manjoo A, Sanders DW, Tieszer C and MacLeod MD: Functional and radiographic results of patients with syndesmotic screw fixation: implications for screw removal. J Orthop Trauma 24(1): 2-6, 2010

26 Jung HG, Kim JI, Park JY, Park JT, Eom JS and Lee DO: Is hardware removal recommended after ankle fracture repair? Biomed Res Int 2016: 5250672, 2016.

27 Brown OL, Dirschl DR and Obremskey WT: Incidence of hardware-related pain and its effect on functional outcomes after open reduction and internal fixation of ankle fractures. J Orthop Trauma 15(4): 271-274, 2001.

28 Hanson B, van der Werken C and Stengel D: Surgeons' beliefs and perceptions about removal of orthopaedic implants. BMC Musculoskelet Disord 9: 73, 2008.
Received October 31, 2018

Revised November 8, 2018

Accepted November 9, 2018 